\title{
Gallbladder Torsion: A Rare Diagnostic Challenge
}

\author{
Charalampos Seretis ${ }^{\mathrm{a}, \mathrm{c}}$, Kasun Wanigasooriya ${ }^{\mathrm{a}}$, Morgan Cleasby ${ }^{\mathrm{b}}$, \\ Raju Tirumularaju ${ }^{a}$, Markos Daskalakis ${ }^{\mathrm{a}}$
}

\begin{abstract}
Gallbladder torsion is a rare cause of acute abdominal pain and can be a diagnostic challenge for the general surgeon. We herein present a case of an 81-year-old man who presented with right-sided abdominal pain and signs of intra-abdominal infection. Transabdominal ultrasound, computed tomography (CT) and magnetic resonance cholangio-pancreatography (MRCP) scans could not identify with certainty the underlying cause of the symptoms and eventually the patient underwent a diagnostic laparoscopy, which revealed the presence of gallbladder torsion. Sequentially, a laparoscopic cholecystectomy was performed in a standard fashion and the patient had an uneventful post-operative recovery. Our case highlights the importance of diagnostic laparoscopy as a diagnostic and treatment tool in unclear cases of acute abdomen.
\end{abstract}

Keywords: Gallbladder; Torsion; Laparoscopy; Surgery

\section{Introduction}

Torsion of the gallbladder is an extremely rare clinical entity, with approximately 500 cases being reported in the international literature up to date [1]. Clinically mimicking acute cholecystitis, gallbladder torsion can be pre-operatively diagnosed incidentally with the use of conventional imaging modalities, namely abdominal ultrasound scan (USS), computed tomography (CT) and magnetic resonance cholangio-pancreatography (MRCP). Frequently the diagnosis is made intra-operatively during cholecystectomy [2-4]. We herein present a case of gallbladder torsion, which was detected intra-operatively during diagnostic laparoscopy for acute diffuse right-sided abdominal

Manuscript submitted March 18, 2020, accepted April 17, 2020

Published online August 22, 2020

aDepartment of General Surgery, Good Hope Hospital, Heart of England NHS Foundation Trust, Sutton Coldfield, Birmingham, UK

bDepartment of Radiology, Good Hope Hospital, Heart of England NHS Foundation Trust, Sutton Coldfield, Birmingham, UK

${ }^{\mathrm{c} C}$ Corresponding Author: Charalampos Seretis, Department of General Surgery, Good Hope Hospital, Heart of England NHS Foundation Trust, Rectory Road, Sutton Coldfield, Birmingham B75 7RR, UK. Email: babismed@gmail.com

doi: https://doi.org/10.14740/jcs405 pain in the absence of definite radiological findings, despite the performance of extensive pre-operative abdominal imaging.

\section{Case Report}

An 81-year-old Caucasian man was referred to our unit with 3-day history of intermittent and non-specific right-sided abdominal pain prior to the admission date. The full set of paraclinical investigations included baseline blood tests, plain chest radiograph and sequential CT scan of thorax, abdomen and pelvis. On admission, the baseline blood test results revealed mild raise of the inflammatory markers and marginal raise of bilirubin (cumulative summary of daily fluctuation of blood test results during hospitalization presented in Table 1 with normal institutional reference values included). Plain radiograph was also unremarkable. Due to the persistence of the clinical symptoms, the patient underwent a CT scan of the thorax, abdomen and pelvis, which demonstrated the presence of focal thickening of the right side of an otherwise normal gallbladder, without presence of biliary tree dilatation (Fig. 1). In view of these results, the patient was treated conservatively as presumed biliary colic and provisionally discharged with a follow-up USS of the abdomen on outpatient basis.

The patient re-attended the emergency department $24 \mathrm{~h}$ post discharge with increasing abdominal pain and fast atrial fibrillation. Repeat blood tests revealed significantly raised white cell count and de-arranged liver function tests with normal amylase and serum lactate (detailed values in Table 1). After initial resuscitation, the patient was commenced on wide spectrum antibiotics and a USS of the abdomen was carried out, which demonstrated the presence of a grossly distended and thickened gallbladder, free fluid in the right perihepatic space and mild intrahepatic biliary dilatation with a common bile duct diameter measured at $7 \mathrm{~mm}$. Of note, no evidence of biliary lithiasis was identified. Moreover, an approximately $4 \mathrm{~cm}$ collection in the lesser sac was seen. In view of the new findings, an MRCP was performed with the clinical impression of extrahepatic lithiasis of the biliary tree resulting in ascending cholangitis. Interestingly, the MRCP failed to detect the presence of lithiasis within the gallbladder or the biliary tree. However, it was noted that the amount of the pericholecystic fluid had increased compared to the previous CT scan, along with progression of the thickening of the gallbladder wall, particularly in the neck of the gallbladder (Figs. 2, 3). 
Table 1. Fluctuation of Blood Test Results During Hospitalization

\begin{tabular}{lllllll}
\hline Parameter & $\begin{array}{l}\text { Initial } \\
\text { admission }\end{array}$ & $\begin{array}{l}\text { Re-attend- } \\
\text { ance date }\end{array}$ & $\begin{array}{l}\text { Second day of } \\
\text { hospitalization }\end{array}$ & $\begin{array}{l}\text { Opera- } \\
\text { tion date }\end{array}$ & $\begin{array}{l}\text { Second post- } \\
\text { operative day }\end{array}$ & $\begin{array}{l}\text { Institutional } \\
\text { normal range }\end{array}$ \\
\hline White cell count & 9.7 & 20.83 & 14.66 & 13.55 & 10.83 & $(4.0-11.0) \times 10^{9}$ \\
Neutrophil count & 7.41 & 17.77 & 12.74 & 11.14 & 8.72 & $(1.5-4.0) \times 10^{9}$ \\
Hemoglobin & 115 & 129 & 107 & 110 & 96 & $135-180 \mathrm{~g} / \mathrm{L}$ \\
C-reactive protein & 47 & - & - & 154 & 130 & $0-5 \mathrm{mg} / \mathrm{L}$ \\
Alkaline phosphatase & 59 & 206 & 122 & 106 & 111 & $30-130 \mathrm{IU} / \mathrm{L}$ \\
Alanine transaminase & 16 & 190 & 98 & 67 & 82 & $0-55 \mathrm{IU} / \mathrm{L}$ \\
Bilirubin & 28 & 37 & 24 & 22 & 17 & $<21 \mu \mathrm{mol} / \mathrm{L}$ \\
Amylase & 57 & 29 & - & 24 & 21 & $-25 \mathrm{IU} / \mathrm{L}$ \\
Plasma lactate & - & 1.4 & - & - & - & $0.5-2.2 \mathrm{mmol} / \mathrm{L}$ \\
\hline
\end{tabular}

Interestingly, on sequential clinical examinations, Murphy's sign was negative and the abdominal tenderness was gradually becoming more marked towards the right iliac fossa rather than the right upper quadrant. In absence of a clear diagnosis despite the thorough imaging, a decision was made to proceed with a diagnostic laparoscopy after informed consent of the patient was obtained. Intra-operatively, upon entry in the peritoneal cavity, a significant amount of purulent fluid was seen in the right iliac fossa and right paracolic gutter. Further inspection of the peritoneal cavity revealed the presence of a gangrenous gallbladder, with the macroscopic necrotic appearances mainly affecting the gallbladder fundus and body, relatively sparing the infundibulum and the gallbladder neck (Fig. 4). Surprisingly, during the manipulation of the gallbladder, it was noted that the gallbladder was very mobile, being attached to the liver via a thin and long connective tissue band (Fig. 5). The clinical diagnosis of gallbladder torsion was established and a laparoscopic cholecystectomy was performed in a standard fashion. After exposure of the hepatocystic triangle, the cystic duct and artery were identified and critical view of safety was achieved. Both the cystic artery and cystic duct were divided between $10 \mathrm{~mm}$ metallic clips (× 2 clips on cystic duct and cystic artery remnants

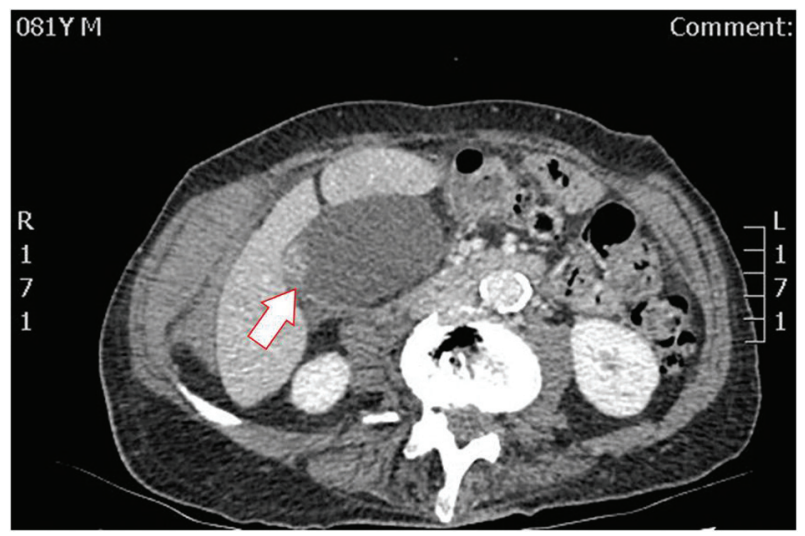

Figure 1. CT scan on admission day demonstrating enlargement of the gallbladder and focal thickening of the gallbladder wall. CT: computed tomography. respectively). The gallbladder body was dissected with hood diathermy off the liver with no concerns regarding hemostasis and/or bile leak. Copious washout in the perihepatic space, right paracolic gutter and pelvis was performed till effluent was clear. A non-suction drain was inserted through the $5 \mathrm{~mm}$ right hypogastric port and was placed in the subhepatic space. The patient had an uneventful recovery on the ward and his abdominal drain was removed on the second postoperative day. He was discharged on the third post-operative day with a course of oral antibiotics and was reviewed in the outpatient clinic 2 months after the operation, with absence of any delayed complications.

\section{Discussion}

Since its initial report by Wendel in 1898 , gallbladder torsion still remains a rare clinical entity $[5,6]$. In terms of underlying mechanism, it appears that the presence of either a complete but long mesentery or an incomplete mesentery covering only the cystic duct and artery can both result in this "floating" appearance of the gallbladder and predispose to future torsion [7]. Both clockwise and anti-clockwise torsion can occur, pre-

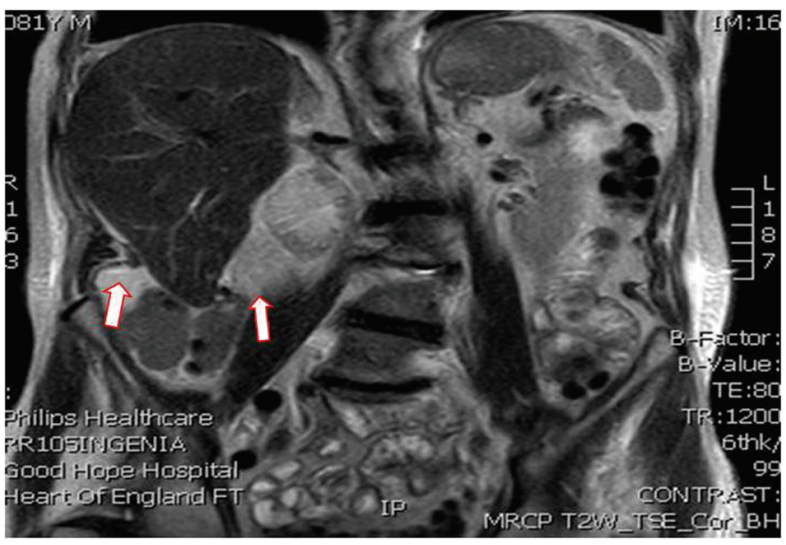

Figure 2. T2-weighted MRCP demonstrating further extension of the perihepatic collection. MRCP: magnetic resonance cholangio-pancreatography. 


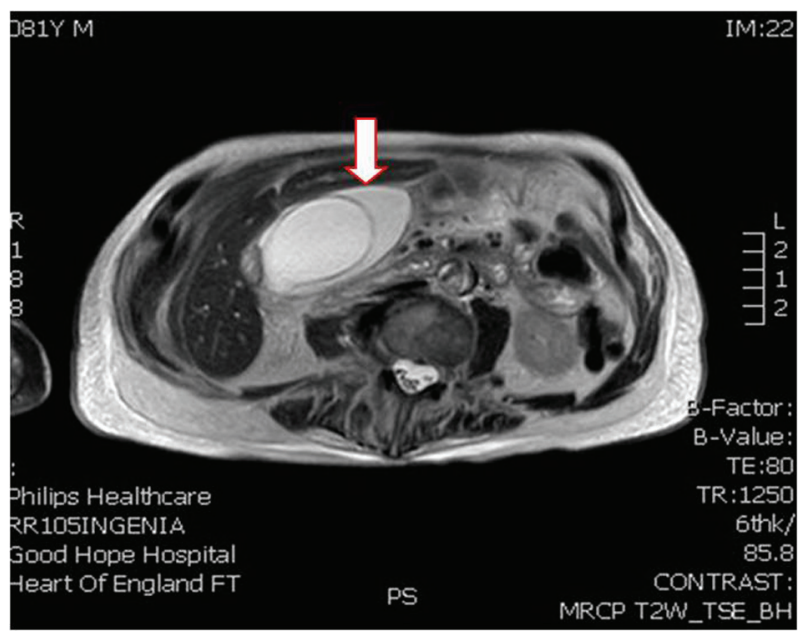

Figure 3. T2-weighted MRCP demonstrating further extension of the perihepatic collection and absence of biliary tree dilatation. MRCP: magnetic resonance cholangio-pancreatography.

sumably as a result of either gastroduodenal or colonic peristalsis respectively [8].

Reilly et al have previously published a systematic review on gallbladder torsion, reviewing the international literature [9]. In a general overview, it appears that gallbladder torsion in the adult population is more frequent in women (approximately $80 \%$ predominance), in contrast to the pediatric population, where the authors describe an approximately $70 \%$ male predominance; mean age of presentation was at an average of 77 years. Interestingly, in the same paper it is stated that only in $20 \%$ of patients the gallbladder torsion was accurately diagnosed pre-operatively, when reviewing only the cases published over the last 20 years. Therefore, it seems that despite the significant advances in the field of abdominal imaging, gallbladder torsion remains an unexpected intra-operative finding.

In accordance with the above, the final diagnosis in our case was established during the performance of the diagnostic laparoscopy. It should be noted that prior to proceeding with the diagnostic laparoscopy, the patient was thoroughly investigated with abdominal ultrasound, $\mathrm{CT}$ and $\mathrm{MRCP}$, which all failed to detect this rare underlying pathology. Of note, the

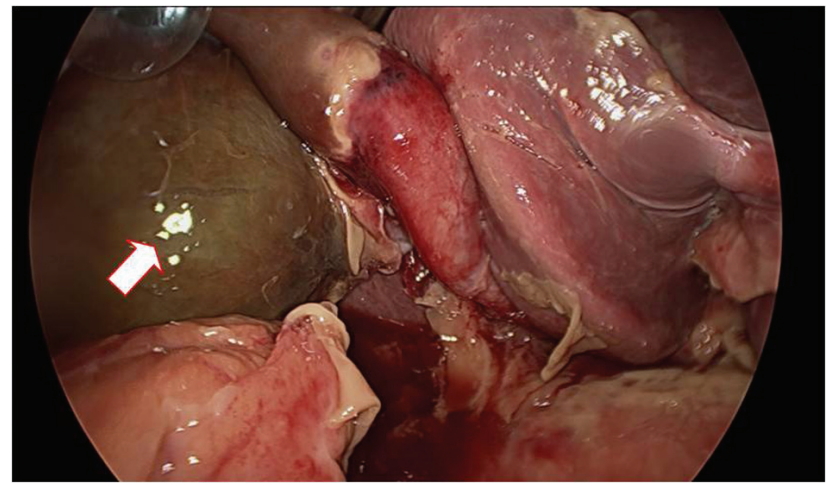

Figure 4. Intra-operative of view of the gallbladder with gangrenous appearance.

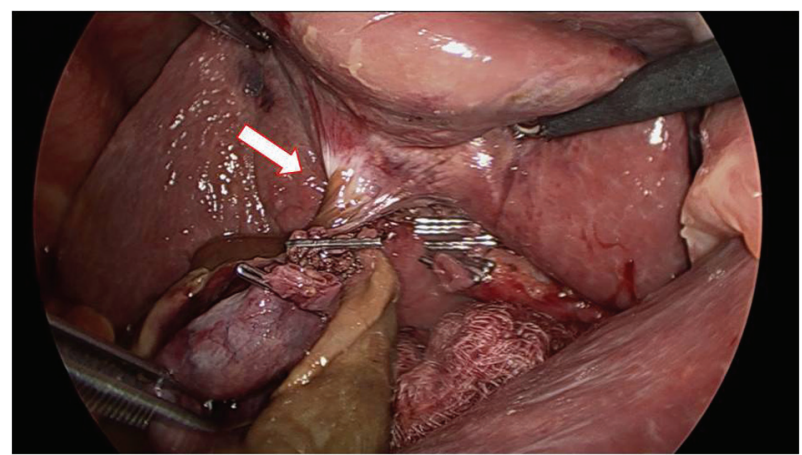

Figure 5. Intra-operative view of the gallbladder torsion around its attachments to the liver after the dissection of the hepatocystic triangle.

inability of the standard pre-operative imaging essays to detect the diagnosis of gallbladder torsion in a patient with suspected cholecystitis has been a common theme in previously published cases [10]. Therefore, it appears that after the exhaustion of the conventional diagnostic means, the value of diagnostic laparoscopy is inappreciable and prompt surgical intervention can result in excellent clinical outcome in this uncommon clinical case.

\section{Acknowledgments}

None to declare.

\section{Financial Disclosure}

None to declare.

\section{Conflict of Interest}

None to declare.

\section{Informed Consent}

We hereby confirm that informed consent was obtained from the patient for publication of the case details.

\section{Author Contributions}

$\mathrm{CS}$ and $\mathrm{KW}$ contributed to the literature research and writing of manuscript. MC, TR, and MD contributed to the editing and reviewing of the manuscript.

\section{Data Availability}

The authors declare that data supporting the findings of this study are available within the article. 


\section{References}

1. Yokoi T, Miyata K, Yuasa N, Takeuchi E, Goto Y, Miyake H, Kobayashi Y, et al. Twisted cystic artery disclosed by 3-dimensional computed tomography angiography for torsion of the gallbladder. Am J Surg. 2011;201(5):e33-34.

2. Koyanagi T, Sato K. Complete gallbladder torsion diagnosed with sequential computed tomography scans: a case report. J Med Case Rep. 2012;6:289.

3. Pu TW, Fu CY, Lu HE, Cheng WT. Complete body-neck torsion of the gallbladder: a case report. World J Gastroenterol. 2014;20(38):14068-14072.

4. Gabizon S, Bradshaw K, Jeyarajan E, Alzubaidy R, Liew V. Gallbladder torsion: a diagnostic challenge. Case Rep Surg. 2014;2014:902814.

5. Wendel AV. VI. A Case of Floating Gall-Bladder and Kid- ney complicated by Cholelithiasis, with Perforation of the Gall-Bladder. Ann Surg. 1898;27(2):199-202.

6. Yeh HC, Weiss MF, Gerson CD. Torsion of the gallbladder: the ultrasonographic features. J Clin Ultrasound. 1989;17(2):123-125.

7. Boonstra EA, van Etten B, Prins TR, Sieders E, van Leeuwen BL. Torsion of the gallbladder. J Gastrointest Surg. 2012;16(4):882-884.

8. Short AR, Paul RG. Torsion of the gallbladder. Br J Surg. 1934;22:301-309.

9. Reilly DJ, Kalogeropoulos G, Thiruchelvam D. Torsion of the gallbladder: a systematic review. HPB (Oxford). 2012;14(10):669-672.

10. Cui J, Abdullah M, Awan I, Mehanna D. Cholecystitis secondary to gallbladder torsion - A rare case report. Int J Surg Case Rep. 2018;46:13-15. 